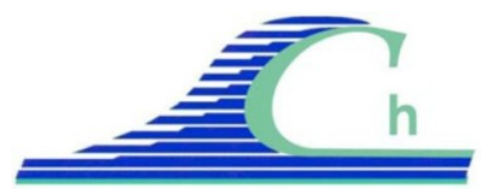

XII ${ }^{\text {ìmes }}$ Journées Nationales Génie Côtier - Génie Civil

Cherbourg, 12-14 juin 2012

DOI:10.5150/jngcgc.2012.016-T @ Editions Paralia CFL

disponible en ligne - http://www.paralia.fr - available online

\title{
Inversion de l'asymétrie de la marée, sur un cycle morte-eau vive-eau, dans l'estuaire de la Charente (France)
}

\author{
Florence TOUBLANC ${ }^{1}$, Isabelle BRENON ${ }^{1}$, Olivier LE MOINE ${ }^{2}$
}

1. Université de La Rochelle, UMR 7266 CNRS - LIENSs, 2 rue Olympe de Gouges, 17000 La Rochelle, France.

florence.toublanc@univ-Ir.fr, isabelle.brenon@univ-lr.fr

2. IFREMER-LERPC, Centre de La Tremblade, Ronce-les-bains, 17390 La Tremblade, France.

olivier.le.moine@ifremer.fr

\section{Résumé :}

La compréhension de la dynamique des estuaires représente un enjeu majeur, à la fois pour des raisons environnementales et économiques. La plupart des études menées sur ces problématiques ont été réalisées sur de grands estuaires (Hudson, Etats-Unis; Severn, Royaume-Uni; Yangtze, Chine; Gironde, France, ...). Les systèmes plus petits tels que l'estuaire de la Charente restent quant à eux peu étudiés et compris. L'hydrodynamique de cet estuaire est ici étudiée au moyen de mesures ADCP. L’asymétrie de la marée, phénomène connu en milieu estuarien, présente certaines particularités dans ce milieu macrotidal et peu profond.

\section{Mots-clés :}

Estuaire - Asymétrie de la marée - Hydrodynamique - Mesures ADCP

\section{Abstract:}

Understanding the hydrodynamics of estuaries is a very sensitive issue, for both economic and environmental reasons. Many studies investigating these dynamics have been conducted on major estuaries (Hudson, USA; Severn, UK; Yangtze, China; Gironde, France, ...), whereas smaller systems such as the Charente estuary (Atlantic coast, France) remain poorly understood. This observation is illustrated here by the tidal asymmetry. Although it is a well-known phenomenon, some unusual aspects appear from the Charente estuary study, based on ADCP measurements.

\section{Keywords :}

Estuary - Tidal asymmetry - Hydrodynamics - ADCP measurements

\section{Introduction}

L'asymétrie tidale est un phénomène connu et communément observé en milieu estuarien. Lors de sa propagation dans un estuaire, la marée présente en effet un comportement asymétrique (distorsion non linéaire de l’onde), impactant directement sa dynamique (AUBREY et al., 1985). Ainsi sont définis les estuaires dominés par le flot, et ceux dominés par le jusant. Lorsqu'une embouchure est dominée par le flot, les 
vitesses de courant sont plus élevées lors de cette phase, et la durée du montant (ou flux) est significativement plus courte que la durée du perdant (ou reflux). Le comportement inverse est observé pour un système dominé par le jusant (AUBREY et al., 1985 ; FRIEDRICHS \& AUBREY, 1988). Ce phénomène a une importance considérable en termes de transport de sédiments (ALLEN et al., 1980). Les estuaires dominés par le flot auront tendance à s'emplir de sédiments alors que les systèmes dominés par le jusant auront tendance à expulser les sédiments vers l’océan.

Plusieurs études prouvent qu'un changement de certaines caractéristiques d'un estuaire peut provoquer, sur le long terme, une inversion de la dominance. Par exemple, des changements significatifs sur la géométrie d'un estuaire (section mouillée, bathymétrie, berges, ...) peuvent influencer l'asymétrie de la marée observée. Une réduction importante de la section de passage à l'entrée d'un chenal aura tendance à favoriser l'apparition d'une dominance de flot (BOON \& BYRNE, 1981). Des variations importantes dans la bathymétrie d'un estuaire peuvent aussi entraîner une inversion de l'asymétrie de la marée (BOLLE et al., 2010). L'apparition au cours du temps de grandes vasières a également une influence non négligeable sur la propagation de la marée (FORTUNADO \& OLIVEIRA, 2005). Ainsi, en présence de vasières, une dominance de jusant sera privilégiée. A l'inverse, pour les systèmes soumis à des régimes macrotidaux, la probabilité d’obtenir une dominance de flot sera plus importante.

Dans le cadre de cette étude, une inversion à court terme de l'asymétrie tidale, sur un cycle vive-eau/morte-eau, a été observée dans l'estuaire de la Charente. La compréhension de ce phénomène particulier représente un enjeu important pour l'étude de l'hydrodynamique mais aussi de la dynamique sédimentaire de l'estuaire. Ces questions scientifiques sont elles-mêmes impliquées dans des problématiques plus larges d'ordre économique et sociétal.

\section{Zone d'étude et contexte}

Le secteur d'étude se situe au centre de la façade Atlantique française. La Charente, fleuve peu profond long de 381,4 km, se jette dans l'Océan Atlantique au nord de la baie de Marennes-Oléron. Ce bassin soumis à un régime macrotidal est sous influence d'une marée semi-diurne. L'amplitude de la marée peut atteindre 6,5 m en embouchure, avec un niveau d'eau moyen proche de $5 \mathrm{~m}$. Selon les différents acteurs et usagers de la Charente, la marée dynamique est ressentie à plus de $80 \mathrm{~km}$ de l'embouchure.

L'estuaire (figure 1) est caractérisé par la présence de grandes vasières et un hydrodynamisme faible, menant à des concentrations de sédiments en suspension très importantes. Le type de sédiments rencontré est quasi exclusivement cohésif (diamètre inférieur à $63 \mu \mathrm{m}$ ). Un barrage, situé à Saint-Savinien (47 km de l'embouchure), permet de réguler les inondations dues à la Charente, en cas de crues liées au fleuve ou à la marée. 


\section{XII ${ }^{\text {èmes }}$ Journées Nationales Génie Côtier - Génie Civil \\ Cherbourg, 12-14 juin 2012}

Les activités économiques de cette région sont nombreuses et diversifiées : pêche et culture marine (huîtres, moules), navigation commerciale, activités portuaires à Rochefort ou à Tonnay-Charente, et activités de loisirs et de tourisme liées à la mer et au fleuve. La Charente représente également une source d'eau potable pour la région.

Dans ce contexte, la compréhension de la dynamique de l'estuaire, qui influence les pertuis charentais d'une manière globale (STANISIERE et al., 2006), représente un enjeu important pour ces activités économiques.

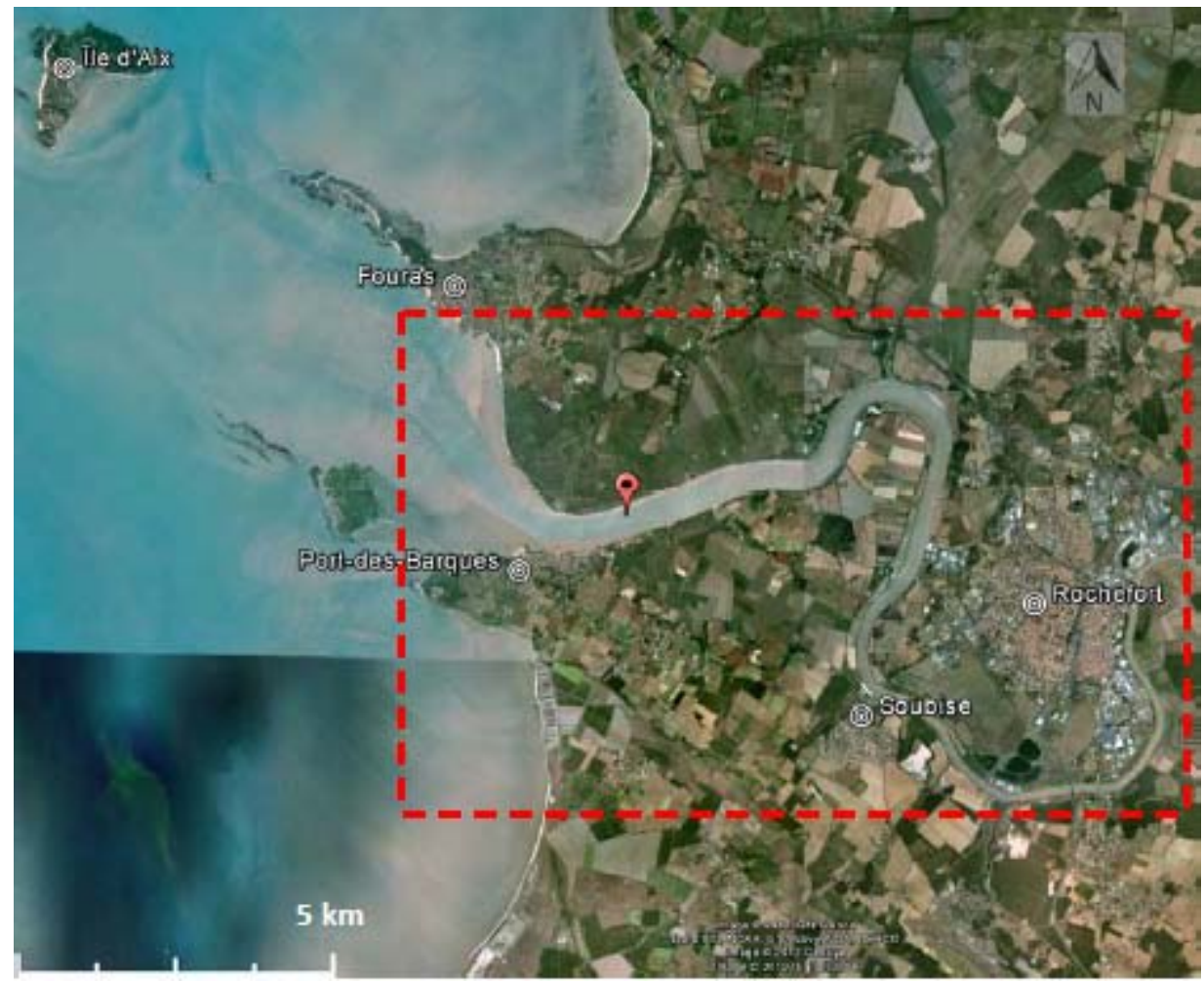

Figure 1. Vue satellite de l'estuaire de la Charente (Google Earth).

\section{Matériel et méthodes}

Cette étude se base principalement sur des mesures effectuées au moyen d'un ADCP (Acoustic Doppler Current Velocimeter). Deux campagnes ont été menées, en 2006 (STANISIERE et al., 2006) et 2011. Le point de mesure en embouchure de Charente (à hauteur de Port des Barques, marqueur rouge sur la figure 1) a été échantillonné pendant des durées de deux mois, en 2006 (du 17 mars au 14 mai) et en 2011 (du 11 février au 8 avril).

Des suivis de débits journaliers sont également utilisés, afin de déterminer le degré d'influence du débit fluvial sur le comportement particulier observé.

A partir des données de hauteur d'eau en fonction du temps, les durées du flot et du jusant ont été calculées. Les données de vitesses ont été intégrées sur la hauteur z, afin d'obtenir une vitesse moyenne en fonction du temps. A partir de ce résultat, les vitesses 
moyennes de flot et de jusant ont été calculées, en omettant les vitesses de la première et de la dernière cellule de mesure (sur 25 cellules au total), celles-ci étant bruitées par l’interface entre les deux milieux.

\section{Résultats et discussion}

Les figures suivantes présentent les résultats obtenus pour la campagne effectuée en 2011. Sur la figure 2, les vitesses moyennes de flot et de jusant sont représentées, en parallèle avec les débits journaliers, et le ratio de ces deux vitesses au cours de la campagne. Les durées de chaque phase de flux ou reflux sont également présentées, avec les données de hauteur d'eau. L’influence du vent est négligée pour cette étude, celui-ci étant faible et peu variant sur toute la durée de la campagne.

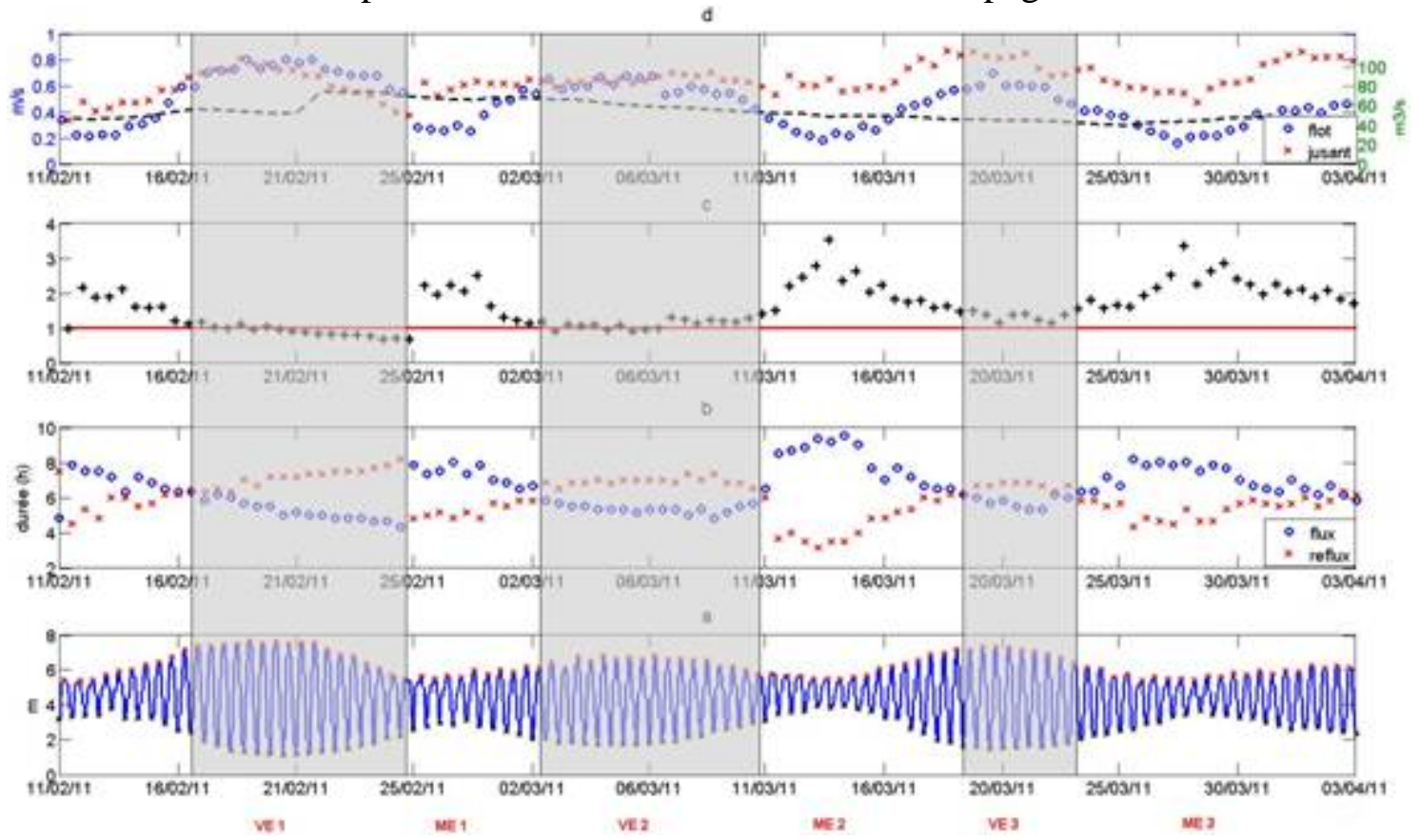

Figure 2. a) Hauteur d'eau, b) Durées flux et reflux (respectivement o et x), c) Ratio vitesses moyennes de flot et de jusant (respectivement o et $x$ ), d) Vitesses moyennes de flot et de jusant, et débit journaliers (traits discontinus).

a) Durées des phases de flux et de reflux

Les durées de flux et de reflux (figure 2b) permettent de mettre en évidence une inversion de l'asymétrie de la marée. Les premières tendances émanant de ces résultats sont les suivantes :

- Lors des périodes de mortes eaux, les durées de reflux sont plus courtes que les durées de flux. Pour les mortes eaux les plus faibles (ME 2), cette tendance est accentuée. La phase de flux est alors trois fois plus longue que la phase de reflux (9h contre 3h). 


\section{XII ${ }^{\text {èmes }}$ Journées Nationales Génie Côtier - Génie Civil \\ Cherbourg, 12-14 juin 2012}

- Lors des périodes de vives eaux, ce sont les phases de flux qui sont plus courtes que les phases de reflux.

Ces résultats ont également été observés sur la campagne menée en 2006. Ces observations suggèrent l'apparition d'une dominance de jusant en morte-eau (zones non grisées, figure 2), et le passage à une dominance de flot en vive-eau (zones grisées).

L'asymétrie tidale remarquée à l'embouchure de la Charente est donc dépendante du moment de la marée. Des résultats similaires avaient été obtenus par LE HIR et al. (2000), sur la vasière de Brouage, située juste au sud de la Charente, et par NICOLLE (2006), en divers endroits des pertuis charentais.

La figure 3 qui représente la hauteur d'eau entre le 8 mars 2011 (VE 2 en déchet) et le 17 mars 2011 (ME 2 en revif), permet d'observer clairement l'inversion de la dominance au cours du temps. Au début de ce signal, le flux est plus court que le reflux. Ce comportement s'inverse entre le 11 mars et le 12 mars, pour aboutir à des phases de reflux plus courtes que les phases de flux pendant les mortes-eaux.

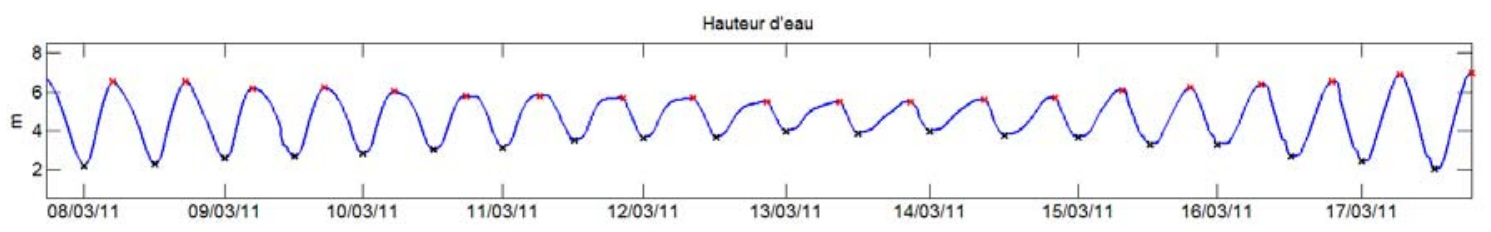

Figure 3. Fin de vive-eau $n^{\circ} 2$ et morte-eau $n^{\circ} 2$.

Cette figure permet de remarquer un autre phénomène : la tenue du plein. Entre le 12 et le 14 mars, la marée montante atteint une sorte de palier à partir duquel l'augmentation de la hauteur d'eau au cours du temps devient très faible, voire inexistante. La figure 4 met en évidence l'occurrence de doubles pleines mers, toujours lors de cette transition entre une dominance de flot et une dominance de jusant (VE 1 vers ME 1). Ces phénomènes, observés dans d'autres estuaires européens (LEVASSEUR et al., 2007), sont très probablement dus à la géométrie particulière des pertuis charentais, qui influence l'hydrodynamique de l'estuaire. Cette géométrie va modifier la propagation de la marée en impactant l'amplification ou non des composantes harmoniques de celleci. Selon MEDEIROS et KJERFVE (1988), l'apparition de doubles pleines mers ou doubles basses mers est due à la superposition d'une marée de fréquence supérieure sur la marée dominante. Les composantes M4 ou M6 peuvent donc venir modifier la propagation attendue de la marée, sous certaines conditions particulières favorisées par des géométries contraintes. C'est le cas des pertuis charentais, avec un trait de côte particulièrement complexe, la présence d'îles et donc de plusieurs chenaux, et enfin une faible profondeur du milieu. La propagation de la marée y est donc directement impactée par ces paramètres (NICOLLE, 2006) 


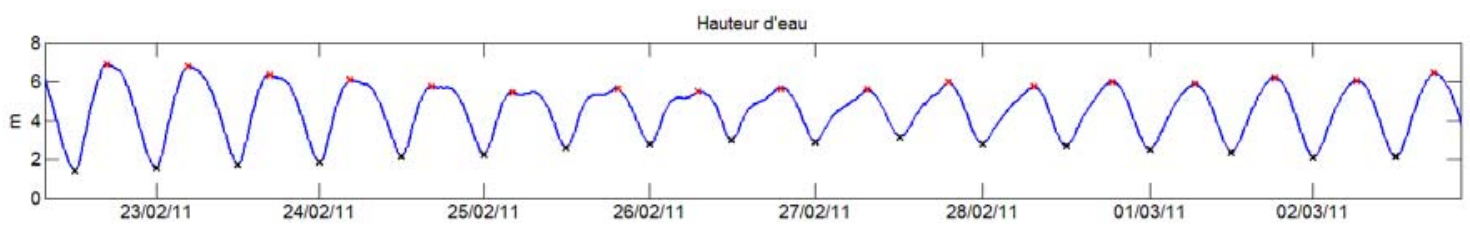

Figure 4. Morte-eau $n^{\circ} 1$.

\section{b) Vitesses moyennes}

L'étude des vitesses moyennes observées pour chaque phase de flux et de reflux permet également de caractériser l'asymétrie de la marée (figure 2d). En effet, dans le cas d'une dominance de jusant, les vitesses devraient être plus élevées pendant le flux par rapport au reflux, et vice versa pour une dominance de flot (FRIEDRICHS \& AUBREY, 1988) :

- Pour les mortes eaux, les vitesses de courant de jusant sont bien plus élevées que les vitesses de courant de flot. De la même manière que pour les hauteurs d'eau, ce comportement est accentué pour les mortes eaux les plus faibles (ME 1).

- Pour les vives eaux, la tendance est moins claire. Les vitesses moyennes de flot sont légèrement plus élevées pour la VE 1 , mais la différence est quasi inexistante pour la VE 2. Lors de la VE 3, les vitesses moyennes de flot restent plus faibles que les vitesses moyennes de jusant.

Ces résultats sont confirmés par les ratios représentés en figure 2c, tous les points supérieurs à 1 représentant des vitesses de jusant plus élevées que les vitesses de flot.

Ces premières observations suggèrent donc qu'une durée de flux plus courte n'entraîne pas systématiquement des vitesses de flot plus élevées (et vice versa).

A partir de ces résultats, l'hypothèse d'un remplissage de l'estuaire en morte-eau, avec des vitesses de flot plus faibles, mais une durée de flux très longue, est envisagée. En vive-eau, les vitesses de jusant restant légèrement plus fortes, ou équivalentes aux vitesses de flot, avec une durée du reflux plus longue, l'estuaire se viderait.

Cette première hypothèse doit cependant être nuancée. Lors des basses mers de vives eaux, il est apparu que l'ADCP ne pouvait pas mesurer de vitesses dans la colonne d'eau, celui-ci étant à peine immergé. Les vitesses lors de ces étales sont donc manquantes dans l'évaluation des vitesses moyennes, ce qui peut biaiser les résultats obtenus. Une deuxième campagne de mesures menée au printemps 2012 permettra donc de confirmer ou non l'hypothèse formulée ci-dessus.

\section{c) Influence du débit}

L’évolution du débit journalier est aussi représentée sur la figure $2 \mathrm{~d}$. Celui-ci varie entre un minimum de $39,7 \mathrm{~m}^{3} / \mathrm{s}$ et un maximum de $74,2 \mathrm{~m}^{3} / \mathrm{s}$, avec une moyenne de $52,5 \mathrm{~m}^{3} / \mathrm{s}$ sur la durée de la campagne.

L’interaction entre le forçage du fleuve et le forçage de la marée peut apporter une première explication au phénomène observé. En morte-eau, le forçage hydrodynamique 


\section{XII ${ }^{\text {èmes }}$ Journées Nationales Génie Côtier - Génie Civil \\ Cherbourg, 12-14 juin 2012}

exercé par le fleuve prendrait en effet le pas sur le forçage tidal, avec pour résultat des phases de reflux plus courtes et des vitesses de courant de jusant plus élevées. En viveeau, le forçage de la marée prendrait le dessus, grâce à des vitesses de courant de marée plus élevées, provoquant un flux plus court.

\section{Conclusions}

Cette étude met en évidence la dynamique particulière d'un estuaire peu profond et soumis à un régime macrotidal tel que l'estuaire de la Charente, concernant l'asymétrie de la marée.

Sur un même cycle de marée, le reflux peut être plus court ou plus long que le flux. En vive-eau, la phase de flux est plus courte, contrairement aux mortes eaux, pour lesquelles la phase de reflux est plus courte. Du point de vue de la durée des phases de flux et de reflux, une inversion de l'asymétrie, sur une courte période de temps, est donc mise en évidence.

Concernant les vitesses moyennes calculées pour chacune des phases, l'inversion de la dominance semble moins claire, puisque les vitesses de jusant sont généralement supérieures ou égales aux vitesses de flot, quelle que soit la période dans le cycle de marée. Une durée de flux ou de reflux n'engendre donc pas nécessairement des vitesses de courant de flot ou de jusant plus élevées au court de ces périodes.

Selon la littérature (FRIEDRICHS \& AUBREY, 1988; WANG et al., 2002), les estuaires peu profonds auraient tendance à être majoritairement dominés par le flot, alors que les estuaires les plus profonds seraient préférentiellement dominés par le jusant. L'estuaire de la Charente est considéré comme peu profond, et présente pourtant une dominance de jusant marquée en morte-eau, et une dominance de flot en vive-eau. Les modélisations numériques hydrodynamiques et hydrosédimentaires de l'estuaire de la Charente sur MARS3D permettront de mettre en évidence les mécanismes pouvant expliquer cette inversion de l'asymétrie de la marée. Plus particulièrement, l'influence des composantes harmoniques de la marée, la forme et la bathymétrie du chenal seront étudiés. Les données de hauteur d'eau à Port des Barques seront également comparées aux mesures marégraphiques issues de la station de l'île d'Aix (réseau RONIM), située à quelques kilomètres de distance.

\section{Remerciements :}

Les auteurs remercient le Conseil Général de la Charente Maritime pour le financement de la thèse dans laquelle cette étude s'inscrit. Le CNRS et l'Université de La Rochelle sont également remerciés. Cette étude n'aurait pas été possible sans le concours du laboratoire Ifremer LERPC de La Tremblade et de la région Poitou Charentes. Christophe Arnaud, Florence Cornette, Jean-Michel Chabirand, Philippe Geairon, Stéphane Robert et Jean-Luc Seugnet sont particulièrement remerciés pour leur aide précieuse sur le terrain. 


\section{Références bibliographiques}

ALLEN G.P., SALOMON J.C., BASSOULLET P., DU PENHOAT Y., DE GRANPRE C. (1980). Effects of tides on mixing and suspended sediment transport in macrotidal estuaries. Sedimentary Geology, n²6 (1-3), pp. 69-90. doi:10.1016/0037-0738(80)90006-8

AUBREY D.G., SPEER P.E. (1985). A study of non-linear tidal propagation in shallow inlet/estuarine systems, Part 1: Observations. Estuarine Coastal and Shelf Science, $\mathrm{n}^{\circ} 21$ (2), pp 185-205. doi:10.1016/0272-7714(85)90096-4

BOLLE A., WANG Z.B, AMOS C., DE RONDE J. (2010). The influence of changes in tidal asymmetry on residual transport in the Western Scheldt. Continental Shelf Research, $n^{\circ} 30$ (8), pp 871-882. doi:10.1016/j.csr.2010.03.001

BOON J.D. III, BYRNE R.J. (1981). On basin hypsometry and the morphodynamic response of coastal inlet systems. Marine Geology, $\mathrm{n}^{\circ} 40$ (1-2), pp 27-48. doi:10.1016/00253227(81)90041-4

FORTUNADO A.B., OLIVEIRA A. (2005). Influence of intertidal flats on tidal asymmetry. Journal of Coastal Research, $\mathrm{n}^{\circ} 21$ (5), pp 1062-1067. doi:10.2112/03-0089.1 FRIEDRICHS C.T., AUBREY D.G. (1988). Non-linear tidal distortion in shallow wellmixed estuaries: a synthesis. Estuarine Coastal and Shelf Science, $\mathrm{n}^{\circ} 27$ (5), pp 521-545. doi:10.1016/0272-7714(88)90082-0

LE HIR P., ROBERTS W., CAZAILlET O., CHRISTIE M., BASSOULLET P., BACHER C. (2000). Characterization of intertidal flat hydrodynamics. Continental Shelf Research, $n^{\circ} 20$ (12-13), pp. 1433-1459. doi:10.1016/S0278-4343(00)00031-5

LEVASSEUR A., SHI L., WELLS N.C, PURDIE D.A., KELLY-GERREYN B.A. (2007). A three-dimensional hydrodynamic model of estuarine circulation with an application to Southampton Water, UK. Estuarine Coastal and Shelf Science, $\mathrm{n}^{\circ} 73$ (34), pp 753-767. doi:10.1016/j.ecss.2007.03.018

MEDEIROS C., KJERFVET B. (1988). Tidal characteristics of the Strait of Magellan. Continental Shelf Research, $n^{\circ} 8$ (8), pp 947-960. doi:10.1016/0278-4343(88)90056-8

NICOLLE A. (2006). Modélisation des marées et des surcotes dans les Pertuis Charentais. Thèse de doctorat, Université de la Rochelle

STANISIERE J-Y., DUMAS F., PLUS M., MAURER D., ROBERT S. (2006). Hydrodynamic characterization of a semi-enclosed coastal system: Marennes-Oléron (France) basin. http://archimer.ifremer.fr/doc/2006/rapport-2353.pdf, Ifremer report.

WANG Z.B, JEUKEN M.C.J.L, GERRITSEN H., DE VRIEND H.J, KORNMAN B.A. (2002). Morphology and asymmetry of the vertical tide in the Westerschelde estuary. Continental Shelf Science, $\mathrm{n}^{\circ} 22$ (17), pp 2599-2609. doi:10.1016/S0278-4343(02)00134-6 